\title{
BMJ Open Aspirin use in central retinal arterial occlusion to prevent ischaemic stroke: a retrospective cohort study in Taiwan
}

\author{
Eugene Yu-Chuan Kang, ${ }^{1,2}$ Yun-Hsuan Lin, ${ }^{2,3}$ Nan-Kai Wang, ${ }^{1,2,4}$ Ling Yeung, ${ }^{2,3}$ \\ Caesar Luo, ${ }^{5}$ Wei-Chi Wu, ${ }^{1,2}$ Chi-Chin Sun, ${ }^{2,3,6}$ Je-Ho Kang, ${ }^{7}$ Ming-Jui Hung, ${ }^{2,8}$ \\ Tien-Hsing Chen ${ }^{2,6,8}$
}

\begin{abstract}
To cite: Kang EY-C, Lin Y-H, Wang $\mathrm{N}-\mathrm{K}$, et al. Aspirin use in central retinal arterial occlusion to prevent ischaemic stroke: a retrospective cohort study in Taiwan. BMJ Open 2019;9:e025455. doi:10.1136/ bmjopen-2018-025455

- Prepublication history and additional material for this paper are available online. To view these files, please visit the journal online (http://dx.doi. org/10.1136/bmjopen-2018025455).
\end{abstract}

EY-CK and Y-HL contributed equally.

Received 16 July 2018 Revised 12 December 2018 Accepted 17 December 2018

Check for updates

(c) Author(s) (or their employer(s)) 2019. Re-use permitted under CC BY-NC. No commercial re-use. See rights and permissions. Published by BMJ.

For numbered affiliations see end of article.

Correspondence to

Dr Tien-Hsing Chen;

skyheart0826@gmail.com

\section{ABSTRACT}

Objective To understand the efficacy of aspirin use for preventing ischaemic stroke after central retinal artery occlusion (CRAO).

Design The retrospective cohort study was conducted using the National Health Insurance Research Database from 1998 to 2013.

Setting A population-based study.

Participants A total of 9437 participants with newly diagnosed CRAO were identified. Participants who had a previous stroke and/or retinal vascular occlusion, were aged $<20$ years and used aspirin 3 months before the event were excluded. There were 3778 eligible participants matched by propensity score, and they were divided into aspirin $(n=434)$ and aspirin-naive $(n=1736)$ groups after the matching.

Methods Cox proportional hazard models and cumulative survival curves were used to assess ischaemic stroke in the study groups, along with log-rank tests to compare group differences.

Main outcome measures Incidence of ischaemic stroke in the aspirin and aspirin-naive groups 1 year after CRA0. Results Of the 3778 patients with newly diagnosed CRA0, 151 (4\%) had a subsequent ischaemic stroke within 1 year. The risk was especially high during the first week of the CRA0. No difference between the aspirin and aspirin-naive groups was found in risk of ischaemic stroke, haemorrhagic stroke, gastrointestinal bleeding, major bleeding, acute coronary syndrome, retinal vein occlusion, new-onset glaucoma, undergoing panretinal photocoagulation or all-cause mortality. Risk factors for ischaemic stroke within 1 year of CRAO included male gender $(p=0.031 ; H R=1.46)$ and age $(p=0.032 ; H R=1.14)$. Conclusions Aspirin use after a CRAO showed no benefit on attenuating the risk of ischaemic stroke. The risk of ischaemic stroke was increased after CRAO especially during the first week. Male gender and age were risk factors for ischaemic stroke after CRAO.

\section{INTRODUCTION}

Since central retinal artery occlusion (CRAO) was first known in 1859, it has been one ocular emergency with many ocular complications and poor visual outcomes. ${ }^{2}$ The typical presentation of CRAO is sudden onset

\section{Strengths and limitations of this study}

- This is the first study analysing immediate aspirin use after central retinal artery occlusion (CRAO) to prevent ischaemic stroke.

- The population provided sufficient subjects with $\mathrm{CRAO}$, which is a rare event, to conduct a propensity score matching.

- The data set provided comprehensive information of aspirin prescription.

- The lack of examination data limited the study to clarify visual outcome or disease severity.

of painless visual loss caused by a sudden stoppage of arterial blood supply to the retina. ${ }^{3}$ The incidence of CRAO is low, approximately 1.5 in 100000 patient-years, so randomised prospective studies on its treatment are difficult to conduct. ${ }^{4}{ }^{5}$ Therefore, there is no guideline-endorsed or agreed-upon treatment available for CRAO. ${ }^{36}$

CRAO was described as a risk factor for ischaemic stroke, coronary artery disease, critical carotid disease and peripheral arterial occlusive disease. ${ }^{78}$ An increased incidence of stroke after all-cause retinal artery occlusion was found using a nationwide data set sample in Taiwan, and an increased incidence of stroke was observed even 0-3 years after the occlusion. ${ }^{9}$ Park et $a l$ and French et al demonstrated a significantly increased incidence of ischaemic stroke after CRAO in Korea and the USA, especially during the first and second week of the CRAO event. ${ }^{1011}$ In addition to an increased risk of ischaemic stroke, the CRAO itself was thought to be an ocular analogue of ischaemic cerebral stroke. Therefore, immediate preventive treatment such as antiplatelet therapy for ischaemic stroke is crucial after CRAO. ${ }^{3}$ CRAO can be divided into two groups: arteritic CRAO and non-arteritic CRAO (NA-CRAO). ${ }^{12}$ Antiplatelet therapy alone may not be considered 
a treatment for arteritic CRAO as it is always caused by giant cell arteritis. For current research, we did not classify the CRAO type, because arteritic CRAO accounts for only $4.5 \%$ of CRAO cases. $^{2}$

Aspirin, one of the most commonly used antiplatelet medication, has been recommended for the secondary prevention of recurrent ischaemic stroke in patients who have had a prior transient ischaemic attack or ischaemic cerebral infarction. ${ }^{1314}$ However, as a primary prevention of ischaemic stroke, aspirin has remained controversial for patients with different comorbidities or gender. ${ }^{13} 1516$ For example, aspirin has not been recommended for the primary prevention of ischaemic stroke in patients with atrial fibrillation (AF) and has no protective effect on ischaemic stroke in patients with diabetes, yet it could reduce the risk of stroke in diabetic women and myocardial infarction in diabetic men. ${ }^{17} 18$ As for patients with CRAO, aspirin was used in some clinical practices after CRAO events for reducing the risk of subsequent thromboembolic occlusion that could affect other organs. ${ }^{19}$ However, the effect of aspirin on ischaemic stroke prevention after CRAO is unclear.

There is limited research analysing the administration of aspirin in CRAO. Although a loading dose of $600 \mathrm{mg}$ of aspirin was suggested in a textbook, ${ }^{20}$ most recommendations for aspirin use were based on expert opinion. ${ }^{3}$ Therefore, we conducted this population-based study using the National Health Insurance Research Database (NHIRD), which comprises Taiwanese population data, from 1998 to 2013. In the present study, we aimed to augment our understanding of the effect of aspirin in ischaemic stroke prevention after CRAO but also to clarify the additional factors associated with the occurrence of ischaemic stroke in patients who have had a CRAO.

\section{METHODS}

\section{Data source}

The current population-based retrospective cohort study was based on the NHIRD, which contains healthcare data from the Taiwan National Health Insurance (NHI) programme. The strengths of the NHIRD studies have been well discussed and mentioned elsewhere. ${ }^{21} 22$ The Taiwan National Health Research Institute has encrypted all personal information in a data set to ensure the privacy of all participants. There is no way to identify any participant, and no leak of confidential information has been reported. The study was based on a data set from the NHIRD, provided by the Bureau of NHI, Department of Health and managed by the National Health Research Institutes in Taiwan. The interpretation and conclusions contained herein do not represent those of the Bureau of NHI. This study was approved by the Ethics Institutional Review Board of Chang Gung Memorial Hospital (IRB 201700418B1), and it also adhered to the tenet of the Declaration of Helsinki.

\section{Patient enrolment, exclusion criteria and aspirin exposure}

We identified patients had first-ever CRAO as principal diagnosis using the International Classification of Diseases, Revision 9, Clinical Modification (ICD-9-CM) code 362.31, from 1 January 1998 to 31 December 2013 (figure 1). We excluded patients who had a history of CRAO before enrolment and those aged $<20$ years. Furthermore, we excluded patients with a history of stroke (ICD-9-CM codes: 430-437) and retinal vascular occlusion (ICD-9-CM codes: 362.30, 362.32-362.36) within 1 year before the index date. The washout period was 3 months before enrolment, and any patient who had taken aspirin or any other antiplatelet agent (eg, clopidogrel,

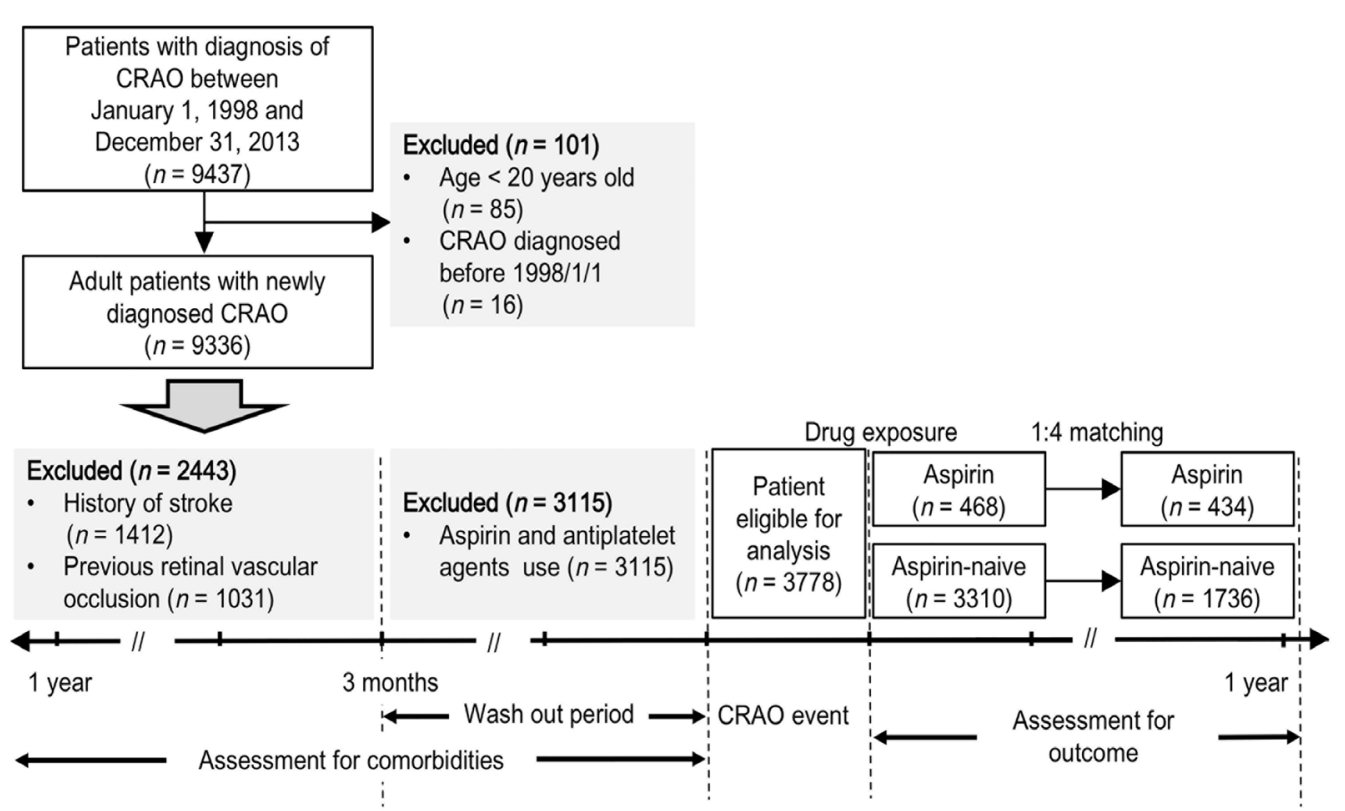

Figure 1 Enrolment of study patients. CRAO, central retinal arterial occlusion. 
ticlopidine and cilostazol) within the washout period was excluded. The remaining eligible cases were separated into aspirin and aspirin-naive groups. Patients in the aspirin group required aspirin administration on the same day of the CRAO diagnosis. Each patient obtained follow-up until the event date, death or 1 year was reached.

\section{Outcome definitions}

The primary outcome was an acute ischaemic stroke (ICD-9-CM codes: 433-437). Acute ischaemic stroke was recognised when the following two criteria were met: (1) hospitalisation; and (2) principal diagnosis of acute ischaemic stroke on admission. Secondary outcomes were safety and ocular outcomes. Safety outcomes analysed in the current study included acute haemorrhagic stroke (ICD-9-CM codes: 430-432), gastrointestinal (GI) bleeding (ICD-9-CM codes: 353-362, 530.21, 530.7, 530.82, 531-534535, 537.83, 537.84, 578), major bleeding events (ICD-9-CM codes: 430-432, 578, 719.1, 423.0, 599.7, $626.2,626.6,626.8,627.0,627.1,786.3,784.7,459.0)$ and all-cause mortality. Mortality was defined if an individual withdrew from the NHI programme; these details of the methodology for estimating safety outcomes and mortality are explained elsewhere. ${ }^{23}$ Ocular outcomes included retinal vein occlusion (ICD-9-CM codes: 327.23 and 362.36), outpatient principal diagnosis of neovascularisation-related glaucoma (ICD-9-CM codes: 365.00-365.06, 365.1, 365.2, 365.61, 365.63, 365.7, 365.81, 365.82, 365.89, and 365.9) and underwent panretinal photocoagulation.

\section{Confounding factors}

An analysis of comorbidities was performed for all patients before matching $(\mathrm{n}=3778)$. Comorbidity was considered if patients had at least two outpatient diagnoses or one inpatient diagnosis 1 year before the index date, as the methodology indicated elsewhere. ${ }^{24}$ Comorbidities investigated in the study included: hypertension (ICD-9-CM codes: 401-405), diabetes mellitus (DM) (ICD-9-CM code: 250), hyperlipidaemia (ICD-9-CM code: 272), chronic kidney disease (CKD) (ICD-9-CM codes: 580-589, 403-404), dialysis (ICD-9-CM codes of catastrophic illness certificate: 585) and AF (ICD-9-CM codes: 427.31 and 427.32). ${ }^{25}$ We also included ocular history such as glaucoma and undergoing panretinal photocoagulation before the index date. Medication use (eg, statin and warfarin) was retrieved from the NHIRD claim data for the 3 months before the index date. Charlson Comorbidity Index (CCI) was recorded based on the scoring system established by Charlson $e t a{ }^{26}$

\section{Statistical analysis}

To minimise the threat of selection bias while comparing outcomes for the aspirin and aspirin-naive groups, we performed a propensity score matching, during which one patient in the aspirin group was matched with four patients in the aspirin-naive group. For the propensity score calculation, covariates included demographic information (eg, age and gender), clinical characteristics (eg, comorbidities, medication and ocular history) and index year of the CRAO occurrence. We compared patient clinical characteristics and demographic information using a $\chi^{2}$ test for categorical variables or two-sample t-test for continuous variables. The risk of outcomes between groups (after matching) was compared using a Cox proportional hazard model in which the study group (aspirin vs aspirin-naive) was the only explanatory variable. We assessed cumulative Kaplan-Meier survival curves for ischaemic stroke against the study groups, along with a log-rank test to compare group differences. We also analysed multivariable Cox models for ischaemic stroke in patients with CRAO before matching to investigate the potential risk factors. Data analysis including propensity score matching was conducted using SAS software, V.9.4 (SAS Institute, Cary, North Carolina, USA).

\section{RESULTS \\ Patient enrolment}

From 1 January 1998 to 31 December 2013, a total of 9437 patients with CRAO were identified. We excluded 85 patients aged $<20$ years, 16 patients diagnosed with CRAO before 1998, 1412 patients with a history of ischaemic stroke, 1031 patients with a history of another retinal vascular occlusion and 3115 patients who used aspirin or any other antiplatelet agent within 3 months of the CRAO event, yielding a total of 3778 patients who were eligible for further analysis. Among the patients eligible for analysis, 468 patients had an aspirin prescription on the same day as the CRAO was diagnosed, and 3310 patients did not. Patients in the aspirin and aspirin-naive groups were propensity score matched based on a 1:4 ratio according to their covariates as previously mentioned. Finally, 434 patients in the aspirin group and 1736 patients in the aspirin-naive group were analysed (figure 1).

\section{Patient and public involvement}

Patients and public were not involved in conducting or in the design of this study.

\section{Incidence of ischaemic stroke}

As for the 3778 patients eligible for analysis before propensity score matching, there were $151(4.0 \%)$ patients who had an ischaemic stroke within 1 year after the CRAO. The number of post-CRAO ischaemic stroke events was stratified by weekly and monthly periods in separated time points (figure 2). The number peaked during the first week. After the first week, the number plunged and gradually decreased in frequency until the end of the first year.

\section{Clinical characteristics of the study cohort}

Clinical characteristics before and after matching are listed in table 1 . In the aspirin and aspirin-naive groups before matching, men represented $62.6 \%$ and $58.5 \%$, respectively, and their average ages were 62.7 and 61.9 years, respectively. In the aspirin and aspirin-naive groups, 


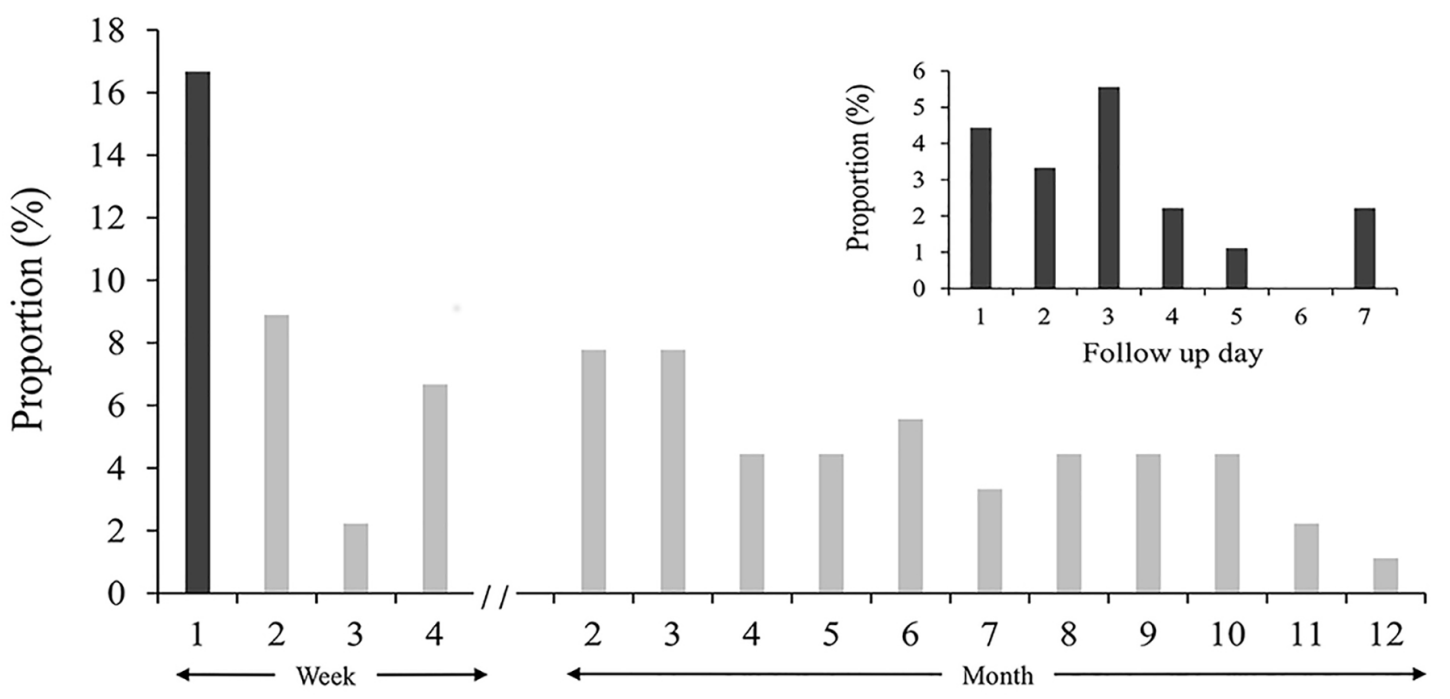

Follow up time

Figure 2 Distribution of stroke occurrence after new-onset central retinal arterial occlusion ( $n=3778)$.

hypertension (29.7\% and $30.5 \%$, respectively) was the most common comorbidity, followed by DM $(13.7 \%$ and $15.6 \%$, respectively) and CKD $(8.5 \%$ and $10.0 \%$, respectively). Further, $62.4 \%$ of the patients in the aspirin group and $60.3 \%$ of patients in the aspirin-naive group did not have any comorbidity before the CRAO. Regarding the history of medication use, $7.3 \%$ and $7.4 \%$ of patients in the aspirin group and the aspirin-naive group had used statin, while only $0.2 \%$ and $0.9 \%$ of patients in the aspirin group and the aspirin-naive group had used warfarin. As for ocular history, $7.7 \%$ of the patients in the aspirin group and $10.1 \%$ of the patients in the aspirin-naive group

Table 1 Clinical characteristics of study cohorts

\begin{tabular}{|c|c|c|c|c|c|c|}
\hline \multirow[b]{2}{*}{ Variables } & \multicolumn{3}{|c|}{ Before matching (\%) } & \multicolumn{3}{|c|}{ After matching (\%) } \\
\hline & $\begin{array}{l}\text { Aspirin } \\
(n=468)\end{array}$ & $\begin{array}{l}\text { Aspirin-naive } \\
(n=3310)\end{array}$ & $P$ value & $\begin{array}{l}\text { Aspirin } \\
(n=434)\end{array}$ & $\begin{array}{l}\text { Aspirin-naive } \\
(n=1736)\end{array}$ & $P$ value \\
\hline Male & $293(62.6)$ & $1938(58.5)$ & 0.095 & 266 (61.3) & $1034(59.6)$ & 0.511 \\
\hline Age (years) ${ }^{*}$ & $62.7 \pm 14.6$ & $61.9 \pm 15.1$ & 0.297 & $62.8 \pm 14.6$ & $62.4 \pm 14.9$ & 0.640 \\
\hline Age $\geq 65$ years & $224(47.9)$ & $1546(46.7)$ & 0.639 & $213(49.1)$ & $849(48.9)$ & 0.949 \\
\hline \multicolumn{7}{|l|}{ Comorbidity } \\
\hline Hypertension & $139(29.7)$ & 1008 (30.5) & 0.740 & 134 (30.9) & $505(29.1)$ & 0.465 \\
\hline Diabetes mellitus & $64(13.7)$ & $517(15.6)$ & 0.275 & $62(14.3)$ & $251(14.5)$ & 0.927 \\
\hline CKD & $40(8.5)$ & $332(10.0)$ & 0.313 & $40(9.2)$ & $145(8.4)$ & 0.564 \\
\hline Hyperlipidaemia & $30(6.4)$ & $228(6.9)$ & 0.701 & $29(6.7)$ & $113(6.5)$ & 0.896 \\
\hline Dialysis & $8(1.7)$ & $100(3.0)$ & 0.111 & $8(1.8)$ & $19(1.1)$ & 0.208 \\
\hline Atrial fibrillation & $3(0.6)$ & $33(1.0)$ & 0.458 & $3(0.7)$ & $9(0.5)$ & 0.664 \\
\hline None of the above & $292(62.4)$ & $1997(60.3)$ & 0.393 & $265(61.1)$ & $1094(63.0)$ & 0.451 \\
\hline \multicolumn{7}{|l|}{ Medication } \\
\hline Statin & $34(7.3)$ & $245(7.4)$ & 0.916 & $32(7.4)$ & 127 (7.3) & 0.967 \\
\hline Warfarin & $1(0.2)$ & $30(0.9)$ & 0.120 & $1(0.2)$ & $3(0.2)$ & 0.802 \\
\hline \multicolumn{7}{|l|}{ Ocular history } \\
\hline Glaucoma & $36(7.7)$ & $334(10.1)$ & 0.102 & $36(8.3)$ & $149(8.6)$ & 0.848 \\
\hline Intervention of PRP & $32(6.8)$ & 275 (8.3) & 0.276 & $31(7.1)$ & $124(7.1)$ & 1.000 \\
\hline CCl score* & $0.72 \pm 1.30$ & $0.85 \pm 1.48$ & 0.074 & $0.77 \pm 1.34$ & $0.72 \pm 1.32$ & 0.476 \\
\hline
\end{tabular}

${ }^{*}$ Presented as mean \pm SD.

$\mathrm{CCl}$, Charlson Comorbidity Index; CKD, chronic kidney disease; PRP, panretinal photocoagulation. 
Table 2 Time to event outcome during a 1-year follow-up

\begin{tabular}{|c|c|c|c|c|}
\hline \multirow[b]{2}{*}{ Outcome } & \multicolumn{2}{|c|}{ Number of events (\%) } & \multicolumn{2}{|c|}{ Aspirin vs Aspirin-naive } \\
\hline & $\begin{array}{l}\text { Aspirin } \\
(\mathrm{n}=434)\end{array}$ & $\begin{array}{l}\text { Aspirin-naive } \\
(n=1736)\end{array}$ & HR (95\% Cl) & $P$ value \\
\hline \multicolumn{5}{|l|}{ Ischaemic stroke } \\
\hline Day 1-7 & $2(0.5)$ & $15(0.9)$ & 0.53 (0.12 to 2.33$)$ & 0.403 \\
\hline Day 1-14 & $4(0.9)$ & $19(1.1)$ & 0.84 (0.29 to 2.47$)$ & 0.752 \\
\hline Day 1-30 & $10(2.3)$ & $23(1.3)$ & 1.74 (0.83 to 3.64$)$ & 0.143 \\
\hline Day $1-90$ & $15(3.5)$ & $37(2.1)$ & 1.63 (0.90 to 2.96$)$ & 0.111 \\
\hline Day $1-180$ & $17(3.9)$ & $50(2.9)$ & 1.37 (0.79 to 2.37$)$ & 0.264 \\
\hline Day 1-365 & $20(4.6)$ & $70(4.0)$ & 1.15 (0.70 to 1.89$)$ & 0.581 \\
\hline \multicolumn{5}{|l|}{ Safety outcome } \\
\hline Haemorrhagic stroke & $1(0.2)$ & $8(0.5)$ & 0.50 (0.06 to 3.99$)$ & 0.513 \\
\hline Gl bleeding & $14(3.2)$ & $60(3.5)$ & 0.93 (0.52 to 1.67$)$ & 0.811 \\
\hline Major bleeding & $5(1.2)$ & $31(1.8)$ & 0.64 (0.25 to 1.65$)$ & 0.359 \\
\hline $\begin{array}{l}\text { Acute coronary } \\
\text { syndrome }\end{array}$ & $1(0.2)$ & $7(0.4)$ & 0.57 (0.07 to 4.64) & 0.600 \\
\hline All-cause mortality & $11(2.5)$ & $47(2.7)$ & 0.93 (0.48 to 1.79$)$ & 0.828 \\
\hline \multicolumn{5}{|l|}{ Ocular outcome } \\
\hline Retinal vein occlusion & $30(6.9)$ & $87(5.0)$ & 1.39 (0.92 to 2.11$)$ & 0.117 \\
\hline New-onset glaucoma & $57(13.1)$ & $184(10.6)$ & 1.26 (0.94 to 1.70$)$ & 0.124 \\
\hline Undergoing PRP & 46 (10.6) & $218(12.6)$ & $0.83(0.60$ to 1.14$)$ & 0.242 \\
\hline
\end{tabular}

GI, gastrointestinal; PRP, panretinal photocoagulation.

had glaucoma, and $6.8 \%$ of the patients in the aspirin group and $8.3 \%$ of the patients in the aspirin-naive group underwent panretinal photocoagulation. The CCI score was 0.72 and 0.85 in the aspirin and aspirin-naive groups, respectively. After matching, there was no significant difference between the aspirin and aspirin-naive groups.

\section{Primary and secondary outcomes after matching}

After matching, the number of event outcomes in the aspirin and aspirin-naive groups is listed in table 2. The mean aspirin dosage in the aspirin group was $93.4 \mathrm{mg}$ / day (data not shown). Among the two groups, there were a total of 90 patients $(4.1 \%)$ who had an ischaemic stroke within 1 year after the CRAO. As for safety outcomes, nine patients $(0.4 \%)$ had a haemorrhagic stroke, 74 patients $(3.4 \%)$ had a GI bleed, 36 patients $(1.7 \%)$ had major bleeding events and eight patients $(0.4 \%)$ had an acute coronary syndrome. The all-cause mortality rate was $2.7 \%$ (58 patients) during this period. As for ocular outcomes, 117 patients $(5.4 \%)$ experienced a subsequent retinal vein occlusion, 241 patients (11.1\%) developed a new-onset neovascularisation-related glaucoma, and 264 patients (12.2\%) underwent panretinal photocoagulation within 1 year. No significant difference was demonstrated between the aspirin and aspirin-naive groups in ischaemic stroke, safety outcomes or ocular outcomes. The Kaplan-Meier survival curves were shown in online supplementary figure 1 to depict ischaemic stroke between the groups. Furthermore, prespecified subgroup analyses were performed, and the observed neutral effect of aspirin was comparable within the subgroups regarding age, gender, DM, hypertension and AF (see online supplementary figure 2).

\section{Risk factor analysis}

We analysed the risk factors for ischaemic stroke within 1 year of CRAO using the population before matching (table 3). Significant risk factors for acute ischaemic stroke were male gender and age (male gender: HR 1.46; 95\% CI 1.04 to 2.05; increasing age: HR 1.14; 95\% CI 1.01 to 1.28). AF might have a trend to be associated with a

Table 3 Risk factor analysis for primary outcomes at a 1-year follow-up before matching $(n=3778)$

\begin{tabular}{|c|c|c|c|}
\hline Predictor & HR & $95 \% \mathrm{Cl}$ & $P$ value \\
\hline \multicolumn{4}{|c|}{ Ischaemic stroke (event number=151) } \\
\hline Male & 1.46 & 1.04 to 2.05 & 0.031 \\
\hline Age (per 10years) & 1.14 & 1.01 to 1.28 & 0.032 \\
\hline Atrial fibrillation & 2.44 & 0.89 to 6.71 & 0.083 \\
\hline Hypertension & 1.26 & 0.88 to 1.82 & 0.211 \\
\hline Diabetes mellitus & 1.13 & 0.71 to 1.78 & 0.607 \\
\hline Hyperlipidaemia & 0.73 & 0.31 to 1.71 & 0.469 \\
\hline $\begin{array}{l}\text { Chronic kidney } \\
\text { disease }\end{array}$ & 0.80 & 0.45 to 1.41 & 0.432 \\
\hline Statin & 1.47 & 0.70 to 3.10 & 0.313 \\
\hline
\end{tabular}


higher risk of ischaemic stroke after CRAO (HR 2.44; $\mathrm{p}=0.083$ ).

\section{DISCUSSION}

To our knowledge, this is the first population-based retrospective cohort study that examined the use of aspirin as a primary prevention for acute ischaemic stroke after CRAO. In this project, immediate aspirin use in patients who had CRAO did not reduce the risk of ischaemic stroke within 1 year. Meanwhile, it had no effect on safety outcomes including haemorrhagic stroke, bleeding events, acute coronary syndrome or all-cause mortality. Aspirin use had neutral effects on ocular outcomes. The risk of ischaemic stroke was highest during the first week. Male patients and older patients had a higher risk of ischaemic stroke within 1 year after the CRAO.

In these patients, CRAO was associated with a risk of stroke, especially during the early stages of a CRAO. ${ }^{810112728}$ Park et al reported an increased risk of ischaemic stroke after CRAO, and the risk was especially high during the first week. ${ }^{10}$ In the USA, French et al also reported that incidence of ischaemic stroke peaked 2 weeks after CRAO. ${ }^{11}$ According to other research, an increased risk was observed up to 3 years after the CRAO event. ${ }^{9}$ Therefore, CRAO might be a predictor of subsequent acute ischaemic stroke, which may have a great impact on quality of life for both patients and their families. An ophthalmologist should focus on not only visual outcomes but also prevention of a subsequent vascular event, especially ischaemic stroke. From our study, the number of patients who experienced an ischaemic stroke was elevated during the first 2 weeks, especially the first 7 days, and gradually decreased after the second week. This finding was compatible with Park and French's investigations. ${ }^{1011}$ This issue affirms the importance of immediate management for stroke prevention after CRAO. ${ }^{29}$

In our study, an add-on strategy involving immediate aspirin use with an average daily dosage of $93.4 \mathrm{mg}$ had no benefit on the primary prevention of ischaemic stroke in patients with CRAO. Aspirin, also known as acetylsalicylic acid, has been commonly used as an antipyretic, analgesic and anti-inflammatory agent. ${ }^{30}$ It reduces the occurrence of atherosclerotic cardiovascular disease (ASCVD) events because its antithrombotic properties can effectively suppress platelet aggregation. ${ }^{14}$ As previously discussed, aspirin use is recommended for secondary prevention of ischaemic stroke based on previous meta-analysis and randomised trials. ${ }^{13} 14$ Moreover, as for the treatment of CRAO, most early studies focused mainly on the reperfusion of the ischaemic retina after CRAO, but the role of aspirin in stroke prevention after CRAO has rarely been investigated. ${ }^{3}$ Park et al indicated that the window of time for stroke prevention is very short, especially during the first week of CRAO, ${ }^{10}$ yet aspirin may still have an effect, as its onset takes only $45 \mathrm{~min}$, and its impact lasts for about 10 days. ${ }^{281}$ In this population-based cohort study, aspirin had no benefit as an immediate primary prevention of ischaemic stroke in patients with CRAO. Although the generally agreed-upon aspirin dose remains controversial for CRAO, ${ }^{320}$ the daily aspirin dose for recurrent stroke prevention is 75 to $100 \mathrm{mg}$ /day, as recommended by the American College of Chest Physicians Evidence-Based Clinical Practice Guidelines. ${ }^{32}$ In this study, in the aspirin group, during the visit when CRAO was diagnosed, the mean aspirin dose was $93.4 \mathrm{mg} /$ day, which met the guideline. However, aspirin therapy with this dose failed to reduce ischaemic stroke risk after patient experiencing CRAO in our study.

Although aspirin is effective for secondary prevention of recurrent ischaemic stroke, it is not suitable for stroke prevention in all situations. For example, using aspirin as monotherapy for primary ischaemic stroke prevention in patients with AF is not recommended. ${ }^{1733}$ According to the latest European guideline, aspirin can only be used as part of a combination therapy for patients with $\mathrm{AF}^{34}$ Instead of aspirin, an anticoagulant such as warfarin or novel oral anticoagulants was recommended in the latest studies. ${ }^{34-36}$ A possible reason for the major modification of medication use was that AF-related ischaemic stroke is caused by thromboembolic events instead of an ASCVD. ${ }^{37}$ Aspirin is effective for the prevention of ASCVD rather than thromboembolic events. ${ }^{14}$ According to earlier papers, the behaviour of CRAO is more similar to thromboembolic diseases than an ASCVD. ${ }^{16} 12$ This could explain why the add-on strategy involving immediate aspirin use failed to prevent an ischaemic stroke in patients with CRAO.

The cumulative risk of stroke for all patients in the study cohort was $0.9 \%$ and $4.0 \%$ at 30 days and 1 year, respectively. As for safety and secondary outcomes, aspirin use did not increase the risk of haemorrhagic stroke, gastrointestinal bleeding or major bleeding in 1 year. Further, it did not affect the risk of common ocular complications after CRAO or all-cause mortality. A subgroup analysis was performed to evaluate the risk of ischaemic stroke in patients with different characteristics, but no difference between the aspirin and aspirin-naive groups was found in any characteristic including age, gender, DM, hypertension and AF. Previous studies have shown that a retinal artery occlusion had similar systemic risk factors for ischaemic stroke, including hypertension, DM and hyperlipidaemia. ${ }^{10}$ In our study, we investigated the additional 1-year risk of ischaemic stroke after CRAO. We found that male gender and age were associated with the occurrence of ischaemic stroke after a CRAO event. Although AF did not reach statistical significance, a trend of increased risk of ischaemic stroke was observed. These findings suggest that CRAO is an early warning sign of ischaemic stroke for patients with risk factors, and efforts to prevent a subsequent stroke and cardiovascular events, including a thorough cardiovascular examination and an immediate referral, should be performed as soon as possible after a CRAO occurrence. ${ }^{29}$

This study had several strengths. First, this is the first cohort study that tested the hypothesis regarding an 
add-on strategy involving immediate aspirin use for the prevention of ischaemic stroke in patients with CRAO. Although it is not a randomised controlled trial, it still could provide information for ophthalmologists to select possible medications to help avoid vascular events after CRAO. Second, this population provided numerous subjects with CRAO, and it allowed us to match the two groups by propensity score. In the real world, obtaining information on a sufficient number of patients for medication trials would have been difficult to accomplish, particularly for the patient group because of the low incidence of CRAO. Third, Taiwan NHIRD provides information on prescription medications. In our project, the use of aspirin, which is only available by prescription, could be obtained, and the dosage could be accurately calculated. Given these strengths, our investigation may be a close representation of an actual patient being treated with aspirin after a CRAO.

There were some limitations in the current study. First, the NHIRD coding system was based on ICD-9 CM codes that do not indicate information on diseases for which physicians provided care. For example, ICD-9 CM codes usually do not classify disease severity. For ophthalmic research, ophthalmologists may expect visual outcome such as visual acuity which NHIRD did not contain. However, we mainly focused on the occurrence of subsequent outcomes after CRAO, and visual outcomes from CRAO are usually poor. In Dr Hayreh's investigation, the initial visual acuity was counting fingers or worse in $93.2 \%$ of the patients with NA-CRAO, and the vision improved in only $9 \%$ of the patients 30 days after the event. ${ }^{2}$ The visual outcomes did not affect our study result. Second, the study did not perform any validation to determine accuracy of the diagnosis based on ICD-9-CM codes. However, more than 2000 articles were published through 2015 using the NHIRD. ${ }^{22}$ Major outcomes in this study such as ischaemic stroke and covariates such as diabetes and hypertension have already been validated. ${ }^{38}{ }^{39}$ Hence, the results of the present study should be faithful. Third, the NHIRD did not contain examination results such as blood tests or images. Regarding CRAO, readers may want to understand the association between carotid artery stenosis or atherosclerosis and subsequent ischaemic stroke, yet we were not able to analyse this issue. Although a carotid ultrasonography was suggested for patients with an incident CRAO and large artery atherosclerosis was found to be associated with retinal artery occlusion, ${ }^{72840}$ some of the previous studies found no association between a retinal embolus and carotid artery stenosis. ${ }^{412}$ In addition, the effect of aspirin used in our study may not be affected whether result of blood test or image examination is available or not.

\section{CONCLUSION}

In conclusion, this is the first study analysing the effect of aspirin after CRAO based on a 16-year population-based data. An add-on strategy involving immediate aspirin use showed no benefit on attenuating the risk of ischaemic stroke, and it did not increase the risk of bleeding events. An elevated risk of ischaemic stroke after CRAO was observed especially during the first 7 days. Risk factors for an ischaemic stroke within 1 year of a CRAO were male gender and age.

\section{Author affiliations}

${ }^{1}$ Department of Ophthalmology, Chang Gung Memorial Hospital, Linkou Medical Center, Taoyuan, Taiwan

${ }^{2}$ Chang Gung University, College of Medicine, Taoyuan, Taiwan

${ }^{3}$ Department of Ophthalmology, Chang Gung Memorial Hospital, Keelung, Taiwan

${ }^{4}$ Edward S. Harkness Eye Institute, Department of Ophthalmology, Columbia University, New York, NY, USA

${ }^{5}$ Bay Area Retina Associates, Oakland, CA, USA

${ }^{6}$ Biostatistical Consultation Center, Chang Gung Memorial Hospital, Keelung, Taiwan

${ }^{7}$ Department of Internal Medicine, Yang Ming Hospital, Taoyuan, Taiwan

${ }^{8}$ Department of Cardiology, Chang Gung Memorial Hospital, Keelung, Taiwan

Contributors Conception and design: YC-K, Y-HL, T-HC; data collection: AC-CS, $\mathrm{M}-\mathrm{JH}$; analysis and interpretation: N-KW, LY, CL, W-CW; writing: Y-CK, Y-HL; revision and document editing: J-HK; overall responsibility: T-HC.

Funding This work was supported by a Chang Gung Memorial Hospital Research Grant, Taoyuan, Taiwan (CGRPG2F0011).

Competing interests None declared.

Patient consent for publication Not required.

Provenance and peer review Not commissioned; externally peer reviewed.

Data sharing statement The data used for the current study cannot be made publicly available according to the NHIRD regulations of personal data protection, allowing only the person responsible for the data management to approach the data after approval from the Taiwan NHI bureau.

Open access This is an open access article distributed in accordance with the Creative Commons Attribution Non Commercial (CC BY-NC 4.0) license, which permits others to distribute, remix, adapt, build upon this work non-commercially, and license their derivative works on different terms, provided the original work is properly cited, appropriate credit is given, any changes made indicated, and the use is non-commercial. See: http://creativecommons.org/licenses/by-nc/4.0/.

\section{REFERENCES}

1. Hayreh SS, Podhajsky PA, Zimmerman MB. Retinal artery occlusion: associated systemic and ophthalmic abnormalities. Ophthalmology 2009;116:1928-36.

2. Hayreh SS, Zimmerman MB. Central retinal artery occlusion: visual outcome. Am J Ophthalmol 2005;140:376.e1-376.e.

3. Fraser SG, Adams W. Interventions for acute non-arteritic central retinal artery occlusion. Cochrane Database Syst Rev 2009:CD001989.

4. Leavitt JA, Larson TA, Hodge DO, et al. The incidence of central retinal artery occlusion in Olmsted County, Minnesota. Am J Ophthalmol 2011;152:820-3.

5. Park SJ, Choi NK, Seo KH, et al. Nationwide incidence of clinically diagnosed central retinal artery occlusion in Korea, 2008 to 2011. Ophthalmology 2014;121:1933-8.

6. Varma DD, Cugati S, Lee AW, et al. A review of central retinal artery occlusion: clinical presentation and management. Eye 2013;27:688-97.

7. Callizo J, Feltgen N, Pantenburg S, et al. Cardiovascular risk factors in central retinal artery occlusion: Results of a prospective and standardized medical examination. Ophthalmology 2015;122:1881-8.

8. Lavin P, Patrylo M, Hollar M, et al. Stroke risk and risk factors in patients with central retinal artery occlusion. Am J Ophthalmol 2018;9394:30479-3.

9. Chang YS, Jan RL, Weng SF, et al. Retinal artery occlusion and the 3-year risk of stroke in Taiwan: a nationwide population-based study. Am J Ophthalmol 2012;154:645-52.

10. Park SJ, Choi NK, Yang BR, et al. Risk and risk periods for stroke and acute myocardial infarction in patients with central retinal artery occlusion. Ophthalmology 2015;122:2336-43. 
11. French DD, Margo CE, Greenberg PB. Ischemic Stroke Risk in Medicare Beneficiaries with Central Retinal Artery Occlusion: A Retrospective Cohort Study. Ophthalmol Ther 2018;7:125-31.

12. Hayreh SS. Ocular vascular occlusive disorders: natural history of visual outcome. Prog Retin Eye Res 2014;41:1-25.

13. Baigent C, Blackwell L, Collins R, et al. Aspirin in the primary and secondary prevention of vascular disease: collaborative metaanalysis of individual participant data from randomised trials. Lancet 2009;373:1849-60.

14. Ittaman SV, VanWormer JJ, Rezkalla SH. The role of aspirin in the prevention of cardiovascular disease. Clin Med Res 2014;12:147-54.

15. Fox CS, Golden SH, Anderson C, et al. Update on Prevention of Cardiovascular Disease in Adults With Type 2 Diabetes Mellitus in Light of Recent Evidence: A Scientific Statement From the American Heart Association and the American Diabetes Association. Circulation 2015;132:691-718.

16. McKibben RA, Al Rifai M, Mathews LM, et al. Primary Prevention of Atherosclerotic Cardiovascular Disease in Women. Curr Cardiovasc Risk Rep 2016;10:10:10.

17. Själander S, Själander A, Svensson PJ, et al. Atrial fibrillation patients do not benefit from acetylsalicylic acid. Europace 2014;16:631-8.

18. Zhang C, Sun A, Zhang P, et al. Aspirin for primary prevention of cardiovascular events in patients with diabetes: A meta-analysis. Diabetes Res Clin Pract 2010;87:211-8.

19. Beatty S, Au Eong KG. Acute occlusion of the retinal arteries: current concepts and recent advances in diagnosis and management. $J$ Accid Emerg Med 2000;17:324-9.

20. Bowling B. Retinal Vascular Disease :In. Kanski's Clinical Ophthalmology: A Systematic Approach. 8th ed. China: Elsevier, 2016:555.

21. Cheng SH, Chiang TL. The effect of universal health insurance on health care utilization in Taiwan. Results from a natural experiment. JAMA 1997;278:89-93.

22. Hsing AW, loannidis JP. Nationwide Population Science: Lessons From the Taiwan National Health Insurance Research Database. JAMA Intern Med 2015;175:1527-9.

23. Wu CY, Chen YJ, Ho HJ, Cy W, Hj H, et al. Association between nucleoside analogues and risk of hepatitis $B$ virus-related hepatocellular carcinoma recurrence following liver resection. JAMA 2012;308:1906-14.

24. Hsu CY, Lin MS, Su YJ, Yj S, et al. Cumulative immunosuppressant exposure is associated with diversified cancer risk among 14832 patients with systemic lupus erythematosus: a nested case-control study. Rheumatology 2017;56:620-8.

25. Chen SW, Chang CH, Lin YS, et al. Effect of dialysis dependence and duration on post-coronary artery bypass grafting outcomes in patients with chronic kidney disease: A nationwide cohort study in Asia. Int J Cardiol 2016;223:65-71.

26. Charlson ME, Pompei P, Ales KL, et al. A new method of classifying prognostic comorbidity in longitudinal studies: development and validation. J Chronic Dis 1987;40:373-83.
27. Lee J, Kim SW, Lee SC, et al. Co-occurrence of acute retinal artery occlusion and acute ischemic stroke: diffusion-weighted magnetic resonance imaging study. Am J Ophthalmol 2014;157:1231-8.

28. Douglas DJ, Schuler JJ, Buchbinder D, et al. The association of central retinal artery occlusion and extracranial carotid artery disease. Ann Surg 1988;208:85-90.

29. Biousse V, Nahab F, Newman NJ. Management of Acute Retinal Ischemia: Follow the Guidelines!. Ophthalmology 2018:125:1597-607.

30. Aspirin: Drug information. UpToDate $®$ Topic 8907 Version 195.0. https://www.uptodate.com/ (Retrieved 17 Apr 2017).

31. Awtry EH, Loscalzo J, Aspirin LJCirculation 2000;101:1206-18.

32. Lansberg MG, O'Donnell MJ, Khatri P, et al. Antithrombotic and thrombolytic therapy for ischemic stroke: Antithrombotic therapy and prevention of thrombosis, 9th ed: American college of chest physicians evidence-based clinical practice guidelines. Chest 2012;141:e601S-36.

33. Hart RG, Pearce LA, Aguilar MI. Meta-analysis: antithrombotic therapy to prevent stroke in patients who have nonvalvular atrial fibrillation. Ann Intern Med 2007;146:857-67.

34. Kirchhof P, Benussi S, Kotecha D, et al. ESC Guidelines for the management of atrial fibrillation developed in collaboration with EACTS. Europace 2016;2016:1609-78.

35. Sterne JA, Bodalia PN, Bryden PA, et al. Oral anticoagulants for primary prevention, treatment and secondary prevention of venous thromboembolic disease, and for prevention of stroke in atrial fibrillation: systematic review, network meta-analysis and costeffectiveness analysis. Health Technol Assess 2017;21:1-386.

36. Hart RG, Pearce LA, Koudstaal PJ. Transient ischemic attacks in patients with atrial fibrillation: implications for secondary prevention: the European Atrial Fibrillation Trial and Stroke Prevention in Atrial Fibrillation III trial. Stroke 2004;35:948-51.

37. Doufekias E, Segal AZ, Kizer JR. Cardiogenic and aortogenic brain embolism. J Am Coll Cardiol 2008;51:1049-59.

38. Cheng CL, Kao YH, Lin SJ, et al. Validation of the National Health Insurance Research Database with ischemic stroke cases in Taiwan. Pharmacoepidemiol Drug Saf 2011;20:236-42.

39. Cheng CL, Lee $\mathrm{CH}$, Chen PS, et al. Validation of acute myocardial infarction cases in the national health insurance research database in taiwan. J Epidemiol 2014;24:500-7.

40. Hong JH, Sohn SI, Kwak J, et al. Retinal artery occlusion and associated recurrent vascular risk with underlying etiologies. PLOS One 2017;12:e0177663

41. Wong TY, Klein R. Retinal arteriolar emboli: epidemiology and risk of stroke. Curr Opin Ophthalmol 2002;13:142-6.

42. Sharma S, Brown GC, Pater JL, et al. Does a visible retinal embolus increase the likelihood of hemodynamically significant carotid artery stenosis in patients with acute retinal arterial occlusion? Arch Ophthalmol 1998;116:1602-6. 